\title{
IMPACTO DE UNA INTERVENCIÓN EN SALUD TRANSCULTURAL LIDERADA POR ADOLESCENTES AUTÓCTONOS E INMIGRANTES SOBRE LA ACTIVIDAD FÍSICA DE NIÑOS Y NIÑAS DE EDUCACIÓN PRIMARIA
}

\author{
Encarnación Soriano \& Verónica C. Cala \\ Universidad de Almería \\ https://doi.org/10.17060/ijodaep.2017.n1.v1.903
}

Fecha de Recepción: 1 Enero 2017

Fecha de Admisión: 1 Abril 2017

\section{RESUMEN}

La conformación de sociedades multiculturales obliga a definir nuevos enfoques educativos en salud que comprendan las similitudes y diferencias culturales de los grupos socioculturales que conviven, reconociendo sus potencialidades y sus distintas problemáticas.

El objetivo de este estudio reside en conocer los efectos sobre la actividad física de una intervención educativa en salud transcultural liderada por alumnado de secundaria español, marroquí y rumano -en calidad de mediadores culturales en salud- sobre alumnado de primaria.

Método. Estudio cuasiexperimental con grupo experimental y control con el alumnado participante de sexto de Educación Primaria.

Instrumentos. Escala de evaluación de las actitudes hacia la actividad física. Resultados. Se aprecian niveles de actividad física superiores en la población marroquí respecto a los otros dos grupos culturales. Tras la intervención se valora una mejoría en los conocimientos sobre los beneficios de la actividad física y un aumento de la motivación referida. A nivel actitudinal se produce un incremento en las actitudes hacia la actividad física marginalmente significativo en hombres del grupo experimental, tanto de Educación Primaria como de Educación Secundaria.

Palabras claves. Educación para la salud transcultural, intervención, actividad física, adolescentes inmigrantes

\section{ABSTRACT}

The formation of multicultural societies requires the definition of new educational approaches in understanding of the similarities and cultural differences of socio-cultural groups that coexisting, recognizing their potentialities and their different problems.

The objective of this study is to know the effects on the physical activity of an intervention education program in cross-cultural health led by Spanish, Moroccan and Romanian - as cultural mediators in health - on primary school students.

Method. Quasiexperimental study with experimental group and control with the participant students of sixth of Primary Education. 


\section{IMPACTO DE UNA INTERVENCIÓN EN SALUD TRANSCULTURAL LIDERADA POR ADOLESCENTES AUTÓCTONOS E INMIGRANTES SOBRE LA ACTIVIDAD FíSICA DE NIÑOS Y NIÑAS DE EDUCACIÓN PRIMARIA}

Instruments. Scale of evaluation of the attitudes towards the physical activity.

Results. High levels of physical activity in the Moroccan population are to the other two cultural groups. After the intervention, an improvement in the knowledge on the benefits of physical activity and an increase in motivation. At the Attitudinal Level there is an increase in attitudes toward marginally significant physical activity in men of the experimental group, both Primary Education and Education High school.

Keywords: transcultural health education; intervention physical activity; adolescents; immigrants

\section{INTRODUCCIÓN}

En los últimos años ha habido un aumento de los estilos de vida sedentarios que han ido acompañados de la disminución de la actividad física diaria especialmente entre en la población joven y adolescente (Tercedor, y otros, 2007). Los análisis realizados en diferentes provincias españolas reflejan que no se cumplen los mínimos establecidos por la OMS, que establece una práctica física de al menos 60 minutos diarios. Así, el estudio de López-Sánchez, González-Víllora, \& Díaz-Suárez (2016) registra un promedio de 60 minutos de ejercicio físico tan solo 3,29 días a la semana y establece que de acuerdo a los estándares el $77 \%$ de los escolares estudiados se puede considerar inac-tivo. Bosque-Lorente \& Baena-Extremera (2014) en su estudio realizado con alumnado de $3^{\circ}$ ESO reconoce que casi un $65 \%$ de los adolescentes afirma practicar actividades físico-deportivas fuera del horario escolar, aunque nuevamente sin alcanzar los niveles que establece la OMS.

La cuestión del ejercicio físico ha cobrado tal importancia en la escuela que la propia LOMCE (2013), ley educativa vigente, añade como novedad en la disposición final un apartado específico de promoción de la actividad física y dieta equilibrada.

Educación e intervenciones en actividad física en contextos multiculturales

Los efectos de las intervenciones en actividad física en la población joven y adolescente han sido sobradamente demostrados. Entre los beneficios de la actividad física destacan su papel preventivo sobre una serie de trastornos y enfermedades como la obesidad y las enfermedades cardiovasculares (Tremblay et al., 2011; OMS, 2010; Mowforth y Daniel, 2007;), una mejora del estado de ánimo, la autoestima, la confianza, el bienestar y la calidad de vida, como forma de prevención de la depresión y la ansiedad y de reducir los niveles de estrés, el consumo excesivo de alcohol, la comodidad y la obesidad (Hassmen et al., 2000, Stanner, 2005).

Sin embargo las experiencias decoloniales, interculturales o transculturales en la intervención física son prácticamente nulas. De hecho, las formas educativas en relación al ejercio físico mantienen huellas coloniales que se expresan en la práctica física. Amusa (2010) explica cómo se extendió por África una educación física colonial, copia exacta de los modelos blancos occidentales, que no atendían a las actividades endémicas o juegos tradicionales sino que se centraban en la imposición del fútbol, criquet, etc. La actitud general que mantenían los gobiernos coloniales hacia los juegos y deportes locales indígenas no fue favorable por lo que tachaban la mayoría de las actividades se consideraban primitivas, inmorales y anticristianas. La única forma de corregir estas prácticas era a través de la educación occidental y por medio de la educación física formalizada- que, para la población indígena, carecía de relevancia.

La educación física colonial guarda similitudes con el tipo de EF impartida en los centros educativos de nuestro entorno, coincidimos con Burnett-van Tonder (1987) que la educación física que se imparte en los centros educativos no se preocupa por los hábitos de los jóvenes racializados, desarrolla fórmulas dependientes del profesorado, con poca variación y centrado en promover el interés individual frente a los valores colectivos que en muchas sociedades y culturas son dominantes.

\section{OBJETIVOS}

El objetivo de este estudio reside en conocer los efectos de una intervención educativa en salud transcultural liderada por alumnado de secundaria español, marroquí y rumano en calidad de mediadores culturales en salud sobre alumnado de sexto de primaria en un contexto multicultural. 


\section{MÉTODO}

La investigación aplica un diseño cuasi-experimental basado en el desarrollo, aplicación y evaluación de una intervención en salud transcultural a través de mediadores culturales en salud, alumnado de educación secundaria previamente formado, sobre alumnado de educación primaria.

De entre los posibles diseños cuasi-experimentales, este proyecto se ha decantado por un diseño pretest-postest con control.

\section{Participantes}

La muestra ha estado formada por 52 alumnos de sexto de Educación Primaria. 26 forman el grupo experimental, del que 15 son hombres y 11 mujeres. 26 forman el grupo control de los cuales 9 son hombres y 17 mujeres.

Tabla 1. Participantes

\begin{tabular}{|c|c|c|c|c|}
\hline & & Grado & Número & Centro \\
\hline Participantes & de & Primaria & 26 participantes $\left(6^{\circ} \mathrm{B}\right)$ & CEIP La Libertad \\
\hline primaria & & ( $6^{\circ}$ primaria) & 26 controles $\left(6^{\circ} \mathrm{A}\right)$ & \\
\hline
\end{tabular}

\section{Descripción de la intervención transcultural en actividad física}

La intervención tuvo un abordaje integral de la salud por lo que una parte considerable de las sesiones estuvieron centradas en la actividad física. Concretamente se buscaba

(1) entender la actividad física como una experiencia social, recreativa, estética, ascética, dirigida al bienestar.

(2) Conocer la diversidad de actividades físicas que existen, tanto en formas de actividad como en variedad cultural.

(3) Pensar los efectos del ejercicio sobre los cuerpos en movimiento (placer, interrelación)

(4) Concebir la actividad como una vía para estrechar lazos entre culturas

Con estos objetivos específicos se desarrollaron 6 sesiones que analizaban las diferencias y similitudes culturales existentes en la práctica de la actividad física, se desarrollaron juegos y danzas de distintas partes del mundo, se profundizó en los efectos físico corporales, psicológicos y sociales del ejercicio físico y se investigó las formas en las que se mediatiza la actividad física a través de los mass media y el impacto de ello.

Para una de las sesiones se contó con jugadores de la Agrupación Deportiva Almería para que trabajasen con mediadores culturales en salud y con alumnado de primaria.

\section{Instrumento}

Se adaptó y validó la escala de evaluación de las actitudes hacia la actividad física de Khan et al. (2012). La estructura de la escala inicial constaba de 24 ítems. (Khan, Asif, Zia-Ul-Islam, Khan, \& Din, 2012). Tras realizar ese proceso de adaptación se mantuvieron 16 items. La escala es tipo Likert y la respuesta va desde totalmente de acuerdo a totalmente en desacuerdo. Ejemplos de Items: Las actividades físicas son una forma de relacionarte con otras personas, La realización de ejercicio ayuda a mejorar tu capacidad de estudio, Los comportamientos negativos se pueden modificar mediante el ejercicio físico, Cuando se participa en actividades físicas se desarrollan movimientos y habilidades creativas. 
Los niveles de confiabilidad de la nueva escala, obtenidos con el método de Alfa de Cronbach, fue de 0, 69 que se encuentra próximo a 0,70.

\section{RESULTADOS}

\section{Descriptivos}

El cuestionario sobre actitudes hacia la actividad física valora el ejercicio en sus distintas dimensiones. La tabla 3 exhibe los valores obtenidos pretest y postest al aplicar la escala sobre alumnado de primaria.

Tabla 2. Puntuaciones del alumnado de sexto de Educación Primaria

\begin{tabular}{|c|c|c|c|c|c|c|c|c|c|}
\hline & & & $\mathrm{N}$ & Media & $\begin{array}{l}\text { Desv. } \\
\text { Estan. }\end{array}$ & & $\mathrm{N}$ & Media & $\begin{array}{l}\text { Desv. } \\
\text { Estan. }\end{array}$ \\
\hline PRETEST & Total & & 50 & 62,80 & 7,97 & & & & \\
\hline \multirow{12}{*}{$\begin{array}{c}\text { Grupo } \\
\text { Experimental }\end{array}$} & Total & & 25 & 62,68 & 7,71 & \multirow{12}{*}{$\begin{array}{l}\text { Grupo } \\
\text { Control }\end{array}$} & 25 & 62,92 & 8,36 \\
\hline & hombre & & 14 & 60,64 & 8,55 & & 9 & 60,11 & 10,21 \\
\hline & & español & 6 & 55,5 & 7,84 & & 3 & 52,00 & 7,55 \\
\hline & & MarroquílG & 3 & 57,33 & 1,53 & & 3 & 71,67 & 4,04 \\
\hline & & Marroqui2G & 4 & 69,50 & 5,91 & & & & \\
\hline & & rumana & 1 & 66,00 & - & & 3 & 56,67 & 5,13 \\
\hline & mujer & & 11 & 65,27 & 5,87 & & 16 & 64,41 & 7,09 \\
\hline & & Española & 4 & 68,75 & 8,02 & & 10 & 61,30 & 5,31 \\
\hline & & MarroquílG & 1 & 61,00 & & & 1 & 76,00 & \\
\hline & & Marroqui2G & 4 & 65,25 & 2,21 & & 4 & 68,50 & 5,32 \\
\hline & & Rumana & 1 & 57,00 & & & 2 & 66,00 & 12,72 \\
\hline & & Senegalesa & 1 & 64,00 & & & & & \\
\hline \multicolumn{2}{|l|}{ POSTEST } & Total & 50 & 65,21 & 8,49 & & & & \\
\hline \multirow{12}{*}{$\begin{array}{c}\text { Grupo } \\
\text { Experimental }\end{array}$} & & & 26 & 66,08 & 9,33 & \multirow{12}{*}{$\begin{array}{c}\text { Grupo } \\
\text { Control }\end{array}$} & 26 & 64,46 & 7,87 \\
\hline & hombre & & 15 & 65,33 & 9,87 & & 9 & 63,67 & 6,50 \\
\hline & & español & 6 & 65,17 & 10,55 & & 3 & 54,33 & 5,50 \\
\hline & & Marroquí $1 \mathrm{G}$ & 4 & 65,00 & 8,36 & & 3 & 71,67 & 4,04 \\
\hline & & Marroqui 2G & 4 & 66,25 & 14,03 & & & & \\
\hline & & rumana & 1 & 64,00 & - & & 3 & 65,66 & 6,51 \\
\hline & mujer & & 11 & 66,81 & 8,48 & & 17 & 64,88 & 7,48 \\
\hline & & Española & 4 & 67,25 & 10,21 & & 10 & 62,10 & 8,19 \\
\hline & & MarroquílG & 1 & 59,00 & & & 1 & 72,00 & \\
\hline & & Marroqui2G & 4 & 72,00 & 6,48 & & 4 & 70,00 & 2,94 \\
\hline & & Rumana & 1 & 58,00 & & & 2 & 65,00 & 5,66 \\
\hline & & Senegalesa & 1 & 51,00 & & & & & \\
\hline
\end{tabular}

A la luz de los datos, se aprecia un incremento de la puntuación pretest-postest de forma que los valores medios pasan de 62,80 a 65,21. La intervención se tradujo en una mejora de las actitudes especialmente en el grupo experimental, se puede ver que el grupo experimental comienza con una puntuación de 62,68 y acaba con 66,08 mientras que el control lo hace con 62,92 y termina con 64,46 . El grupo experimental y control comienzan con puntuaciones muy igualadas que en el postest se ven modificadas. 
En los grupos culturales, destacan las puntuaciones de los jóvenes marroquíes de primera y segunda generación, especialmente aquellos de segunda. Los españoles, al igual que en la escala de alimentación, presentan cifras inferiores en sus puntuaciones -aunque serán los que presenten mayor aumento-.

Por otro lado, el valor promedio es superior en las mujeres que en los hombres, con actitudes más positivas hacia el ejercicio físico. Entre los hombres, las puntuaciones son ligeramente más elevadas en aquellos de origen marroquí, mientras que en las mujeres son algo superiores en las autóctonas.

Para interpretar adecuadamente los valores de la escala, se realizó una adaptación de los criterios de interpretación de las puntuaciones de la escala. Se estableció que los valores de 16 a 32 referían actitudes negativas hacia la actividad física, de los 32 a los 49 una actitud neutral, de los 50 a los 65 una actitud positiva y por encima de los 65 una actitud muy positiva.

Tabla 3. Criterios de interpretación de la escala de actitudes hacia la actividad física (16 items)

\begin{tabular}{cc}
\hline Puntaje en la escala & Significado \\
\hline $16-32$ puntos & Actitud negativa hacia la actividad física \\
$33-49$ puntos & Actitud neutral \\
$50-64$ puntos & Actitud positiva \\
$65-80$ & Actitud muy positiva \\
\hline
\end{tabular}

A partir de los intervalos de referencia, se observa que la mayoría de los grupos sostiene una actitud positiva hacia la actividad física. Ninguno de los jóvenes experimentales o controles, en fase pretest 0 postest, presentó actitudes abiertamente negativas hacia la actividad física. De forma similar, los porcentajes de jóvenes con actitudes neutrales fueron bastante bajos (entre un $0 \%$ y un $8 \%$, por grupos). Por lo que el grueso se ubica fundamentalmente en las actitudes positivas (53,8 y un $64 \%$ ) 0 en las muy positivas menor medida (entre un 32 y un $42,3 \%$ )

Tabla 4. Distribución de los jóvenes del estudio según su nivel de actitud hacia la actividad física.

\begin{tabular}{lcccc}
\hline & Actitud negativa & Actitud neutral & Actitud positiva & Actitud muy positiva \\
\hline Pretest & 0 & $2(4 \%)$ & $31(62 \%)$ & $17(34 \%)$ \\
Experimental & 0 & 0 & $16(64 \%)$ & $9(36 \%)$ \\
Control & 0 & $2(8 \%)$ & $15(60 \%)$ & $8(32 \%)$ \\
Postest & 0 & $3(6 \%)$ & $28(56 \%)$ & $19(38 \%)$ \\
Experimental & 0 & $1(3,85 \%)$ & $14(53,8 \%)$ & $11(42,3 \%)$ \\
Control & 0 & 2 & 14 & $8(33,3 \%)$ \\
\hline
\end{tabular}

\section{Efectividad de la intervención sobre el grupo experimental y control}

Tras la intervención las actitudes hacia el ejercicio físico mantuvieron cambios significativos $(p<0,044)$. El aumento promedio entre ambos momentos fue de tres puntos, de 62,8 a 65,25. El efecto del paso del tiempo se puede considerar bajo ( $d$ de Cohen $=-0,296 ; r=-0,147$ ). 


\section{IMPACTO DE UNA INTERVENCIÓN EN SALUD TRANSCULTURAL LIDERADA POR ADOLESCENTES AUTÓCTONOS E INMIGRANTES SOBRE LA ACTIVIDAD FÍSICA DE NIÑNOS Y NIÑAS DE EDUCACIÓN PRIMARIA}

Tabla 5. Prueba t para muestras emparejadas con el total de la muestra de primaria

\begin{tabular}{|c|c|c|c|c|c|c|c|c|}
\hline & \multicolumn{2}{|c|}{ Medias } & \multicolumn{3}{|c|}{ Diferencias emparejadas } & \multirow[b]{2}{*}{$\mathrm{t}$} & \multirow[b]{2}{*}{$\mathrm{gl}$} & \multirow{2}{*}{$\begin{array}{c}\text { Sig. } \\
\text { (bilateral) }\end{array}$} \\
\hline & Pretest & Postest & Media & $\begin{array}{c}\text { Desviación } \\
\text { estándar }\end{array}$ & $\begin{array}{l}\text { Media de } \\
\text { error est. }\end{array}$ & & & \\
\hline $\begin{array}{l}\text { Actividad física } \\
\text { pretest- postest }\end{array}$ & 62,80 & 65,25 & $-2,45$ & 8,48 & 1,19 & $-2,06$ & 50 & $0,044^{*}$ \\
\hline
\end{tabular}

A diferencia de los resultados generales, es posible deducir que no se han producido cambios estadísticamente significativos en las actitudes hacia la actividad física imputables al papel de la intervención. En el grupo experimental se aprecia un ascenso de los valores que duplica el que se da en el grupo control, que asciende a 1,53.

Tabla 6. Prueba t para muestras emparejadas en grupo experimental y control

\begin{tabular}{|c|c|c|c|c|c|c|c|}
\hline & \multicolumn{5}{|c|}{ Diferencias emparejadas } & \multirow[b]{2}{*}{ gl } & \multirow[b]{2}{*}{$\begin{array}{c}\text { Sig. } \\
\text { (bilateral) }\end{array}$} \\
\hline & Media & Media & $\begin{array}{c}\text { Desviación } \\
\text { estándar }\end{array}$ & $\begin{array}{c}\text { Media de } \\
\text { error est }\end{array}$ & $\mathrm{t}$ & & \\
\hline Grupo Experimental & $\begin{array}{l}62,68 \\
66,08\end{array}$ & $-3,40$ & 9,78 & 1,95 & $-1,73$ & 24 & 0,090 \\
\hline Grupo control & $\begin{array}{l}62,92 \\
64,46\end{array}$ & $-1,53$ & 7,08 & 1,39 & $-1,107$ & 25 & 0,279 \\
\hline
\end{tabular}

Por otra parte, el efecto sobre los distintos grupos culturales no es significativo. Se producen aumentos en la puntuación de todos que no son explicables por su participación o no en el programa. Los españoles del grupo experimental incrementan sus puntuaciones en 5,20 puntos, los marroquíes en 5,75 y los rumanos en 1,75. En el grupo control por su parte, los españoles aumentan 10,15, los marroquíes bajan 0 suben 1,5 puntos según correspondan a $1^{\underline{a}} 02^{\underline{a}}$ generación y los rumanos lo hacen en 5 puntos.

Tabla 7. Prueba t para muestras relacionadas sobre actividad física Pretest-Postest por grupo experimentalcontrol y país de nacimiento

\begin{tabular}{|c|c|c|c|c|c|c|c|c|}
\hline & & \multicolumn{6}{|c|}{ Diferencias emparejadas } & \multirow[b]{2}{*}{$\begin{array}{c}\text { Sig. } \\
\text { (bilateral) }\end{array}$} \\
\hline & & Media & Media & $\begin{array}{l}\text { Desviación } \\
\text { estándar }\end{array}$ & $\begin{array}{l}\text { Media } \\
\text { error } \\
\text { estand }\end{array}$ & $\mathrm{t}$ & $\mathrm{gl}$ & \\
\hline \multirow{4}{*}{ Experimental } & Españoles & $\begin{array}{l}60,80 \\
66,00\end{array}$ & $-5,20$ & 8,91 & 2,81 & $-1,847$ & 9 & 0,098 \\
\hline & & 58,25 & & & & & & \\
\hline & Marroquies IG & 64,00 & $-5,75$ & 9,67 & 4,84 & $-10,19$ & 3 & 0,302 \\
\hline & Marroquíes $2^{\circ} \mathrm{G}$ & $\begin{array}{c}67,37 \\
69,125\end{array}$ & $-1,75$ & 12,87 & 5,55 & $-0,386$ & 7 & 0,711 \\
\hline \multirow{4}{*}{ Control } & Españoles & $\begin{array}{l}59,15 \\
60,31\end{array}$ & $-1,15$ & 8,87 & 2,46 & $-0,47$ & 12 & 0,647 \\
\hline & Marroquies 1G & $\begin{array}{l}72,75 \\
71,25\end{array}$ & 1,50 & 2,38 & 1,19 & 1,260 & 3 & 0,297 \\
\hline & Marroquies $2^{a} \mathrm{G}$ & $\begin{array}{l}68,50 \\
70,00\end{array}$ & $-1,50$ & 2,51 & 1,26 & 1,19 & 3 & 0,319 \\
\hline & Rumanos & $\begin{array}{l}60,40 \\
65,40\end{array}$ & $-5,00$ & 6,63 & 2,97 & $-1,69$ & 4 & 0,167 \\
\hline
\end{tabular}




\section{DISCUSIÓN Y CONCLUSIONES}

\section{Efecto de la intervención en educación física}

Con respecto al efecto de la intervención, se reconoce un incremento en los conocimientos sobre los beneficios del ejercicio físico sobre el cuerpo. En materia de actitudes hacia la actividad física, se distingue que el alumnado de primaria presenta actitudes considerablemente positivas, con unas medias que oscilan entre 62-68 en un rango se extiende de 24 a 80 . Los buenos resultados en EF se explican por la mejor condición física de los jóvenes que viven en el medio rural o semi-rural frente a aquellos que lo hacen en medios urbanos (Casajús, 2012). Esta relación rural-urbana con puntuaciones tan diferenciadas puede tener su base en la escasez de otras formas de ocio en el ámbito rural. Esa falta de alternativas es manifestada, de forma recurrente, por los jóvenes. Además, existe otro tipo de factores característicos, como el carácter colectivo y socializador de los deportes y la práctica fuera de formatos reglados de práctica del deporte (no recurren a federarse en ligas ni en campeonatos).

Por grupos culturales, el alumnado de primaria muestra diferencias significativas estadísticas. Las y los jóvenes procedentes de Marruecos ofrecen actitudes más positivas que los demás grupos. Y, a su vez, los rumanos tienen también puntuaciones más elevadas que las y los de origen español. Los niveles de actividad física en los jóvenes migrantes marroquíes contradicen los estudios realizados en EEUU (Brewer \& Kimbro, 2014; Taverno-Ross, Francis, BeLue, \& Viruell-Fuentes, 2012; Moore, Diez Roux, Evenson, McGinn, \& Brines, 2008), Mexico (Palos-Lucio, y otros, 2015) 0 España (Gonzalo-Almorox \& Urbanos-Garrido, 2016) que sostienen que los jóvenes migrantes presentan niveles inferiores de actividad física. La existencia de actitudes positivas hacia la actividad física en los marroquíes puede estar indirectamente relacionada con la prescripción coránica de comer ligero y cuidar el cuerpo (Romeike, Abidi, Lechner, de Vries, \& Oenema, 2016).

El estudio de Hamrani, y otros (2015) realizado con adolescentes marroquíes de entre 15 y 19 años en Marruecos sostiene que los patrones de actividad física y la intensidad difieren entre géneros. Los adolescentes varones eran más activos que las adolescentes y participaban en actividades físicas de mayor intensidad; mientras que ellas sostenían más tiempo una actividad física de intensidad moderada

Aunque no hay estudios pormenorizados sobre los procesos de aculturación que se producen en los jóvenes migrantes participantes en el proyecto, los resultados parecen contradecir las conclusiones del estudio de Marks et al. (1990) que establece que los procesos de aculturación pueden influir positivamente sobre la práctica del ejercicio físico (a diferencia de otros comportamientos que se ven empeorados como la alimentación o el consumo de alcohol). En nuestro caso, los grupos menos aculturados en la cultura española, son los que más actividad física mantienen. Por lo que, en cierto modo, la westernización de la EF se ve acompañada de mayor sedentarismo. De igual modo, los factores religiosos parecen influir en la realización de ejercicio ligeramente, sobre todo en el caso de las mujeres marroquíes.

Los españoles destacan con puntuaciones inferiores en sus actitudes hacia el ejercicio físico. Los estudios sobre el tema muestran resultados ambivalentes. Así, algunos informes europeos sitúan a los jóvenes españoles en mejor situación que los de otros países europeos (Loyen, y otros, 2016); mientras que análisis realizados en diferentes provincias reflejan que no se alcanzan los mínimos que establece la OMS. Al respecto, el estudio de López-Sánchez, González-Víllora, \& DíazSuárez (2016) presenta un promedio de 60 minutos -3,29 días/semana- por lo que, de acuerdo a los estándares, el $77 \%$ de los escolares estudiados se puede considerar inactivo. En el realizado (Bosque-Lorente \& Baena-Extremera, 2014) con alumnado de 3을 ESO reconocen que casi un 65,0\% de los adolescentes afirma practicar actividades físico-deportivas fuera del horario escolar, aunque muchos sin alcanzar los niveles que establece la OMS. 


\section{IMPACTO DE UNA INTERVENCIÓN EN SALUD TRANSCULTURAL LIDERADA POR ADOLESCENTES AUTÓCTONOS E INMIGRANTES SOBRE LA ACTIVIDAD FÍSICA DE NIÑNOS Y NIÑAS DE EDUCACIÓN PRIMARIA}

\section{Evolución de las actitudes hacia ejercicio físico}

De acuerdo con los criterios de interpretación de la escala, la media global del pretest y el postest reflejan actitudes positivas o muy positivas. Con el desarrollo de la propuesta de intervención se produce un avance actitudinal en la muestra de los alumnos de sexto de primaria, con un aumento de las puntuaciones en el grupo experimental que eleva sus actitudes a muy positivas. En ambos casos, el incremento de actitudes positivas no se explica estrictamente por efecto de la intervención aunque sí participa en ello. El grupo experimental incrementa sus valores de forma marginalmente estadística, pasando de 62,80 a 65,25; mientras que en el control el ascenso no lo hace de forma significativa.

Se produce un aumento significativo de la puntuación general entre el pretest y el postest. En el grupo experimental, los españoles son los que más incrementan de forma marginalmente significativa sus puntuaciones. El hecho de tener una situación de partida muy baja puede explicar su mayor incremento con respecto a otros grupos.

En el caso de la actividad física los jóvenes con mejores actitudes hacia la salud son, a su vez, los que más incrementen sus actitudes. Esto señala la importancia de los procesos de motivación en la realización de deporte y actividad física.

\section{Efecto de las intervenciones por pares}

De acuerdo con Turner (1999), las iniciativas realizadas por pares/iguales y el apoyo recíproco entre los mismos, tienen un efecto beneficioso sobre las habilidades sociales, la confianza y el sentido de auto-eficacia. El autor explica que, en ocasiones, los jóvenes se perciben a sí mismos como receptores pasivos, sin legitimidad ni respaldo en el control de sus vidas y su salud (que casi siempre dependen de las recomendaciones de profesionales). En consecuencia, las iniciativas realizadas por iguales pueden iniciar procesos de desarrollo emocional, personal y social propios. Cabe recalcar una mayor significatividad de las peer-led intervenciones durante la adolescencia, que los estudios de Mellanby, Newcombe, Rees, \& Tripp (2001); Mellanby, Rees, \& Tripp (2000) muestran mucho mejores resultados que las lideradas por adultos sobre sus iguales -adult-led- .

En la adolescencia los amigos e iguales, durante este periodo juegan un papel fundamental a la hora de servir de apoyo mutuo, reforzar comportamientos sanos y ser una fuente de información en la que confiar (Johnson, Simon, \& Eun-Young, 2014). La interacción física real entre jóvenes (no virtual, tal y como demuestran Eysenbach, Powell, Englesakis, Rizo, \& Stern, 2004) tiene una influencia positiva para los mismos. Pero además también tiene otros efectos beneficiosos. El Mentoring Resource Center (2005) considera que este tipo de programas influyen también en la conectividad de los estudiantes a la escuela y los padres, la mejora de las habilidades sociales y la autoestima y el rendimiento académico, y mentores y aprendices se benefician de la participación en los programas. Adicionalmente, aunque es una cuestión todavía controvertida, favorecen la prevención de conductas de riesgo para la salud (MacArthur, Harrison, Caldwell, Hickman, \& Campbell, 2015; Harden, Oakley, \& Oliver, 2001; Mellanby, Rees, \& Tripp, 2000; Harden, Weston, \& Oakley, 1999).

El debate de la efectividad de estos métodos se encuentra en el punto de mira de muchas investigaciones. De ser efectivo, podría suponer un importante ahorro para los ahogados sistemas sanitarios públicos. Por este motivo, una gran batería de estudios de revisión sistemática y metaanálisis han tratado y siguen tratando de abordar esta cuestión. Los resultados son diversos y solo son concluyentes en determinadas áreas 0 con determinados grupos. Existen unas dimensiones de la salud que parecen más sensibles a esta estrategia que otras. A modo de ejemplo, la revisión realizada por Webel, Okonsky, Trompeta, \& Holzemel (2010) afirma que las intervenciones por pares 
adultos facilitan resultados positivos en ejercicio físico, tabaquismo, uso de condón, pero no son significativas en la alimentación de pecho (breastfeeding), adherencia a medicamentos, salud femenina o participación general en actividades.

\section{REFERENCIAS}

Amusa, L. O. (2010). Las Fases de Cambio de la Educación Física y el Deporte en África:¿ Puede Emerger un Único Modelo Africano? Revista Electrónica Actividad Física y Ciencias, 2(1), 1-15.

Bosque-Lorente, M. B., \& Baena-Extremera, A. (2014). Actividad físico-deportiva de los alumnos de tercer ciclo de Primaria y de la ESO en su tiempo libre. Lorente, M. B., \& Extremera, A. B. (2014). Actividad físico-deportiva de los alumnos de tercer Agora para la educación física y el deporte, 16(1), 36-49.

Brewer, M., \& Kimbro, R. T. (2014). Neighborhood context and immigrant children's physical activity. Social Science \& Medicine, 116, 1-9.

Burnett -van Tonder, C. (1987). Die sosio-etniese danse van die Venda-vrou (Trans: The socio -ethnic dances of the Venda woman.) Pretoria: HAUM.

Casajús, J. A. (2012). Condición física, distribución grasa y salud en escolares aragoneses (7 a 12 años). Revista Internacional de Medicina y Ciencias de la Actividad Física y el Deporte, 12(47), 523-537.

Delgado, L., \& Montes, R. (2015). Práctica de actividad física extraescolar y preferencias deportivas en niños preescolares españoles e inmigrantes: Un estudio pilotp. Revista electrónica de terapia ocupacional Galicia, TOG, 20(7).

Eysenbach, G., Powell, J., Englesakis, M., Rizo, C. \& Stern, A. (2004). Health related virtual communities and electronic support groups: Systematic review of the effects of online peer to peer interactions, BMJ, 328 (7449):1166.

Gonzalo-Almorox, E., \& Urbanos-Garrido, R. M. (2016). Decomposing socio-economic inequalities in leisure-time physical inactivity: the case of Spanish children. International. Journal for Equity in Health, 15(106).

Hamrani, A., Mehdad, S., El Kari, K., El Hamdouchi, A., El Menchawy, I., Belghiti, H., \& Mokhtar, N. (2015). Physical activity and dietary habits among Moroccan adolescents. Public health nutrition, 18(10), 1793-1800.

Harden, A., Oakley, A., \& Oliver, S. (2001). Peer-delivered health promotion for young people: a systematic review of different study designs. Health Education Journal, 60, 339-353.

Harden, A., Oakley, A., Weston, R., \& EPI-centre. (1999). A review of the effectiveness and appropriateness of peer-delivered health promotion for young people. London: Institute of Education, University of London.

Hassmen P, Koivula N and Uutela A (2000) Physical exercise and psychological well-being: A population study in Finland. Preventive Medicine 30(1): 17-25.

Hoyo-Lora, M., \& Sañudo Corrales, F. B. (2007). Motivos y hábitos de práctica de actividad física en escolares de 12 a 16 años en una población rural de Sevilla. Revista Internacional de Medicina y Ciencias de la Actividad Física y del Deporte, 2.

Imperial College. (2016). Global Burden of Metabolic Risk Factors of Chronic Diseases. Obtenido de http://www5.imperial.ac.uk/medicine/metabolic_risks/bmi/.

Johnson, V. L., Simon, P., \& Eun-Young, M. (2014). A Peer-Led High School Transition Program Increases Graduation Rates Among Latino Males. The Journal of Educational Research,, 107(3), 186-196. 
Khan, S., Asif, S., Zia-UI-Islam, M., Khan, M. W., \& Din, M. U. (2012). A Study Regarding the Collage Students' Attitudes towards Physical Activities. International. Journal of Academic Research in Business and Social Sciences, 2(9), 189-198.

López-Sánchez, G. F., González-Víllora, S., \& Díaz-Suárez, A. (2016). Level of habitual physical activity in children and adolescents from the Region of Murcia (Spain). SpringerPlus, 5(386).

Lorente, L. M. (2013). La educación para la salud en la escuela como intervención social. Revista Internacional de Ciencias Sociales, 2(1).

Loyen, A., Van Hecke, L., Verloigne, M., Hendriksen, I., Lakerveld, J., Steene-Johannessen, A., y otros. (2016). Variation in population levels of physical activity in European adults according to cross-European studies: a systematic review within DEDIPAC. The International Journal of Behavioral Nutrition and Physical Activity, 13(72).

MacArthur, G. J., Harrison, S., Caldwell, D. M., Hickman, M., \& Campbell, R. (2015). Peer-led interventions to prevent tobacco, alcohol and/or drug use among young people aged 11-21 years: a systematic review and meta-analysis. Addiction, 111(3), 391-407.

Mellanby, A. R., Rees, J. B., \& Tripp, J. H. (2000). Peer-led and adult-led school health education: a critical review of available comparative research. Health Education Research, 15(5), 533-545.

Mellanby, A. R., Newcombe, R. G., Rees, J., \& Tripp, J. H. (2001). A comparative study of peer-led and adult-led school sex education. Health Education Research, 16(4), 481-492.

Moore, L. V., Diez Roux, A. V., Evenson, K. R., McGinn, A. G., \& Brines, S. J. (2008). Availability of recreational resources in minority and low socioeconomic status areas. American Journal of Preventive Medicine, 34, 16-22.

Mowforth, C., \& Daniel, J. (2007). The effect of an acute bout of exercise on body image, mood, and self-efficacy. Health Psychology Update, 16(1/2), 80.

O’Driscoll, T., Banting, L. K., Borkoles, E., Eime, R., \& Polman, R. (2014). A systematic literature review of sport and physical activity participation in culturally and linguistically diverse (CALD) migrant populations. Journal of Immigrant and Minority Health, 16(3), 515-530.

Palos-Lucio, G., Flores, M., Rivera-Pasquel, M., Salgado-de-Snyder, V. N., Monterrubio, E., Henao, S., y otros. (2015). Association between migration and physical activity of school-age children left behind in rural Mexico. International Journal of Public Health, 60(1), 49-58.

Romeike, K., Abidi, L., Lechner, L., Vries, H., \& Oenema, A. (2016). Similarities and differences in underlying beliefs of socio-cognitive factors related to diet and physical activity in lower-educated Dutch, Turkish, and Moroccan adults in the Netherlands: a focus group study. BMC Public Health, 16(1), 813.

Stanner S (ed.) (2005) Cardiovascular Disease: Diet, Nutrition and Emerging Risk Factors. The Report of British Foundation Task Force. London: Blackwell Publishing

Story, M., Lytle, L. A., Birnbaum, A. S., \& Perry, C. L. (2002). Peer led, school based nutrition education for young adolescents: feasibility and process evaluation of the teens study. Journal of School Health, ),. 72(3), 121-127.

Taverno-Ross, S. E., Francis, L. A., BeLue, R. Z., \& Viruell-Fuentes, E. A. (2012). Associations between physical activity and overweight among U.S. youth by immigrant generation: results from the 2007 National Survey of Children's Health. Journal of Physical Activity Health, 9(6), 840-848.

Tercedor, P., Martín-Matillas, M., Chillón, P., Pérez López, I. J., Ortega, F. B., Wärnberg, J., y otros. (2007). Incremento del consumo de tabaco y disminución del nivel de práctica de actividad física en adolescentes españoles: Estudio AVENA. Nutrición Hospitalaria., 22(1), 89-94. 
Tremblay, M. S., Warburton, D. E., Janssen, I., Paterson, D. H., Latimer, A. E., Rhodes, R. E., \& Murumets, K. (2011). New Canadian physical activity guidelines. Applied Physiology, Nutrition, and Metabolism, 36(1), 36-46.

Turner, G. (1999). Peer support and young people's health. Journal of adolescence., 22(4), 567-572.

Webel, A. R., Okonsky, J., Trompeta, J., \& Holzemel, W. L. (2010). A systematic review of the effectiveness of peer-based interventions on health-related behaviors in adults. American Journal of Public Health, 100(2), 247-253.

Wilcox, S., Castro, C., King, A. B., Housemannd, R., \& Brownson, R. C. (2000). Determinants of leisure time physical activity in rural compared with urban older and ethnically diverse women in the United States. Journal of epidemiology and community health, 54(9), 667-672. 
